\title{
Potent antagonism of Escherichia coli, Bacteroides ovatus, Fusobacterium varium, and Enterococcus faecalis, alone or in combination, for enteropathogens in anaerobic continuous flow cultures
}

\author{
T. USHIJIMA AND Y. OZAKI
}

\section{Department of Microbiology, Shiga University of Medical Science, Otsu, Shiga 520-21, Japan}

Summary. Interactions between representative strains of four predominant resident bacteria of the human colon, Escherichia coli, Enterococcus faecalis, Bacteroides ovatus, and Fusobacterium varium, and strains of seven enteropathogens, Yersinia enterocolitica, Shigella flexneri, Salmonella typhimurium, Vibrio parahaemolyticus, $V$. cholerae serogroup non O1, Staphylococcus aureus, and Clostridium perfringens, were examined in studies with an anaerobic continuous flow culture system and medium resembling the content of the mouse caecum (MCM). Potent unilateral antagonism attributable to synergic activities of the resident bacteria against the enteropathogens was evident.

The four resident bacteria persisted at levels of $c .10^{6} \mathrm{cfu} / \mathrm{ml}$ or more in single and in any mixed cultures of the resident species. The seven enteropathogens also persisted in single cultures. In contrast, $Y$. enterocolitica was excluded in several days in mixed cultures with each of the four resident bacteria. Sh. flexneri and Staph. aureus were excluded in the presence of $E$. coli alone. $C$. perfringens, $V$. parahaemolyticus and $V$. cholerae serogroup non $\mathrm{O} 1$ were excluded in the presence of $E$. coli with $B$. ovatus and, in some cases, with additional species. S. typhimurium was the most resistant; only $c$. $10^{2}$-fold reduction of the population level was observed in mixed culture with all four of the resident species. When the amounts of some components in the medium, such as peptone and yeast extract, were increased, $C$.perfringens grew and persisted even in the presence of the four resident bacteria. Sh. flexneri, in contrast decreased steadily, even in enriched media.

\section{Introduction}

Enteropathogens such as species of Shigella, Salmonella, Vibrio, Yersinia or Staphylococcus, which cause diarrhoea in man, do not usually reside in the alimentary canal. Competitive inhibition by the bacteria residing in the canal is considered to be one of the main defence mechanisms against the pathogens. Metabolic end-products of the resident bacteria, such as fatty acids (Maier et al., 1972), bile acids (Tazume et al., 1979), or hydrogen sulphide (Freter et al., 1983a) have been considered to be possible growth-inhibiting agents, but the mechanisms of inhibition remain unclear. In view of the physiological complexity of the canal, efforts to analyse the mechanisms in studies with gnotobiotic

Received 28 Oct. 1985; revised version accepted 10 Dec. 1985. animals have produced results of doubtful validity (Freter et al., 1983b). As populations of the normal faecal flora increase gradually at the lower end of the small intestine and reach a maximum level in the colon, the ileum and colon may be the main sites of bacterial antagonism.

In 1962, Hentges and Freter found that in-vitro antagonisms observed in frequently subcultured or in continuous flow (CF) broth cultures correlated with those noted in vivo. Veilleux and Rowland (1981) and Freter et al. (1983b) also demonstrated similar population levels of bacteria in anaerobic CF cultures to those observed in the lower alimentary canal of conventional or gnotobiotic animals. Accordingly, the anaerobic CF culture system has been considered as an excellent in-vitro model for examining bacterial interactions in lower areas of the alimentary canal. 
Reports of the interactions between the faecal flora and enteropathogens have usually been related to only two or three species (Hentges and Freter, 1962; Maier et al.. 1972; Tazume et al., 1979). One reason for the limitation in the numbers of species studied may be the lack of appropriate selective media for viable counts of each of the co-existing species. We devised selective media (Ushijima et al., 1985) that allow enumeration of 14 or more species of bacteria representative of the normal faecal flora or the enteropathogens. These media made it possible to investigate interactions among several co-existing species in the present study.

\section{Materials and methods}

\section{Bacterial strains}

Enteric pathogens used were Shigella flexneri 3a SU 5031, Salmonella typhimurium LT-2 (wild type), Yersinia enterocolitica SU 5032, Vibrio parahaemolyticus SU 5027. $V$. cholerae (serogroup non 01) SU 5030, Staphylococcus aureus ATCC 25923, and Clostridium perfringens type A VPI 5201. The representatives of the normal faecal flora were Escherichia coli ATCC 25922. ATCC 11775, and some fresh isolates of this species from clinical specimens, Enterococcus faecalis IFO 12969, Bacteroides otatus SU 39. B. fragilis ATCC 25285, B. thetaiotaomicron VPI 2358, Fusobacterium rarium ATCC 8501, and Bifidobacterium adolescentis ATCC 15703. These strains were obtained from the American Type Culture Collection (ATCC), the Anerobe Laboratory, Virginia Polytechnic Institute and State University, Blacksburg, Virginia (VPI), and the Institute for Fermentation, Osaka, Japan (IFO). Some were stock cultures in our laboratory ( $\mathrm{SU}=$ Shiga University).

\section{Culture system and conditions}

The media used for cultivation of bacteria in $\mathrm{CF}$ cultures were a mimic of the contents of the caecum of mice (MCM medium; Ushijima. 1983a) and its modifications. The MCM medium contained (/L) soluble starch (Nakarai Chemical Co. Ltd, Kyoto) 2g, D-glucose 0.5g, $\left(\mathrm{NH}_{4}\right)_{2} \mathrm{SO}_{4} 2 \mathrm{~g}, \mathrm{KH}_{2} \mathrm{PO}_{4}$ (anhydrous) $7 \mathrm{~g}, \mathrm{Na}_{2} \mathrm{HPO}_{4}$ (anhydrous) $10 \mathrm{~g}, \mathrm{NaCl} 2 \mathrm{~g}, \mathrm{Na}_{2} \mathrm{CO}_{3}$ (anhydrous) $1 \mathrm{~g}, \mathrm{MgSO}_{4} \cdot 7$ $\mathrm{H}_{2} \mathrm{O} 0.1 \mathrm{~g}, \mathrm{FeSO}_{4} 7 \mathrm{H}_{2} \mathrm{O} 0.005 \mathrm{~g} . \mathrm{CaCl}_{2}$ (anhydrous) $0.01 \mathrm{~g}$. yeast extract (BBL Microbiology Systems, Cockeysville, MD) $0 \cdot \mathrm{lg}$, Proteose Peptone No. 3 (Difco Laboratories, Detroit, MI) $0.5 \mathrm{~g}$, haemin $0.01 \mathrm{~g}$, vitamin $B_{12} 0.0005 \mathrm{~g}$. vitamin $K_{3} 0.0001 \mathrm{~g}$, Tween $800.25 \mathrm{~g}$, gall powder (Nakarai, Kyoto) $0.05 \mathrm{~g}$, sodium acetate (anhydrous) $5 \mathrm{~g}$. sodium propionate $0.5 \mathrm{~g}$, sodium butyrate (normal) $3 \mathrm{~g}$. sodium lactate $(60 \% \mathrm{v} / \mathrm{v}) 0.5 \mathrm{~g}$, sodium succinate $1 \mathrm{~g}$, sodium palmitate $0.05 \mathrm{~g}$, sodium stearate $0.05 \mathrm{~g}$, sodium linoleate $0.02 \mathrm{~g}$, sodium oleate $0.02 \mathrm{~g}$, sodium thioglycollate $0.2 \mathrm{~g}$, L-cysteine $\mathrm{HCl} . \mathrm{H}_{2} \mathrm{O} \quad 0.5 \mathrm{~g}$, L-aspartic acid sodium salt $0.01 \mathrm{~g}$. L-threonine $0.04 \mathrm{~g}$.
L-serine $0.01 \mathrm{~g}$, L-glutamic acid sodium salt $0.3 \mathrm{~g}$, L-glutamine $0.2 \mathrm{~g}$, L-proline $0.01 \mathrm{~g}$, glycine $0.01 \mathrm{~g}$, L-alanine $0.05 \mathrm{~g}$, L-valine $0.03 \mathrm{~g}$. L-methionine $0.02 \mathrm{~g}$, L-isoleucine $0.01 \mathrm{~g}$, L-tyrosine $0.01 \mathrm{~g}$, L-phenylalanine $0.01 \mathrm{~g}$, L-lysine $\mathrm{HCl} 0.04 \mathrm{~g}$, L-histidine $\mathrm{HCl} 0.01 \mathrm{~g}$, L-arginine $\mathrm{HCl} 0.02 \mathrm{~g}$, and L-tryptophan $0.01 \mathrm{~g}$. The $\mathrm{pH}$ of the medium was adjusted to 7.0 with $\mathrm{HCl} 10 \% \mathrm{v} / \mathrm{v}$ and $\mathrm{NaOH} 10 \% \mathrm{w} / \mathrm{v}$. A stock solution of amino-acid mixture was prepared at a 50-fold concentration and was stored at $4^{\circ} \mathrm{C}$ until use. When abundant gas-forming bacteria such as $C$. perfringens or $F$. varium were included in an experiment, an antifoaming agent, Toray silicone SM 5571 (Toray Silicone Co. Ltd, Tokyo), was added at a final concentration of $100 \mathrm{mg} / \mathrm{L}$.

The anaerobic $C F$ culture system was as described elsewhere (Ushijima, 1984). A diagram of the apparatus is given in fig. 1 . As the culture vessel was designed to avoid a large gas phase over the culture medium, it was unnecessary to flow oxygen-free gas continuously into the vessel to maintain anaerobic conditions. The flow rate of medium was $5 \% / \mathrm{h}(5 \mathrm{ml} / \mathrm{h})$, i.e., fresh broth was added to the vessel to exchange $5 \%$ of the culture per hour.

Inocula were prepared by suspending fresh cultures in GAM semisolid agar medium (Nissui Co. Ltd, Tokyo) in

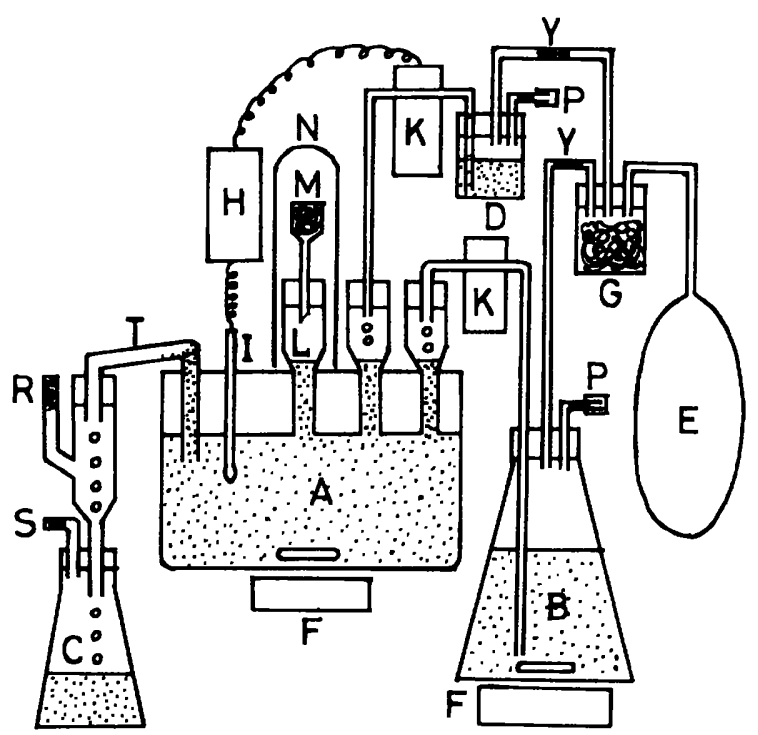

Fig. 1. Schematic figure of an anaerobic continuous flow culture system (Ushijima, 1984). A: growth vessel filled with c. $100 \mathrm{ml}$ of culture medium; excess medium is expelled spontaneously through a siphon (T). B: fresh medium reservoir. C: collecting flask. D: $\mathrm{NaOH}$ or $\mathrm{HCl}$ solution reservoir. E: rubber bladder filled with nitrogen gas. F: magnetic stirrer. $G$ : oxygen-absorbing bottle filled with steel wool treated with $\mathrm{CuSO}_{4} 1 \%$ in water at $p \mathrm{H}$ 2 or less (Parker, 1955). $\mathrm{H}$ : $p \mathrm{H}$ controller. I: $p \mathrm{H}$ glass combination electrode. K: peristaltic pump. L: port for inoculation and sampling of bacteria. M: gas outlet needle with cotton wool plug. $\mathrm{N}$ : plastic cap for preventing contamination of the port. P: nitrogen gas inlet tube with cotton plug. The air of the vessels (B and $D$ ) is expelled by flushing with nitrogen gas and then the tips of the tubes are capped tightly with rubber stoppers. $\mathbf{R}$ and $\mathbf{S}$ : inlet and outlet tubes of gas with cotton wool plug. Y: cotton wool plug to prevent contamination of bottles B and D. 
saline at a concentration equivalent to McFarland No.3-4. A 0.5-ml volume of the suspension of each strain was mixed and inoculated into the vessel. When organisms representing the natural faecal flora were required as inocula, $1 \mathrm{~g}$ of normal adult faeces was suspended in $9 \mathrm{ml}$ of saline and left to stand for $c .5 \mathrm{~min}$. The upper layer $(1 \mathrm{ml})$ was used as inoculum.

The numbers of each test strain co-existing in the vessel were determined every day or every 2 days after inoculation, by viable counts with selective media (Ushijima et $a l ., 1983 b, 1985)$. E. coli strains, other than ATCC 25922 , were usually detected on agar plates of ALPPL medium, without penicillin and lincomycin. $V$. cholerae serogroup non 01 was detected with the $V$. parahaemolyticus selective medium (VPBC). The newly devised selective medium for Sh. flexneri was composed of $(/ \mathrm{L})$ Trypticase Peptone (BBL) 10g, Proteose Peptone No. $33 \mathrm{~g}$, yeast extract 2g, $\mathrm{NaCl} 5 \mathrm{~g}, \mathrm{Na}_{2} \mathrm{HPO}_{4}$ (anhydrous) $4 \mathrm{~g}, \mathrm{KH}_{2} \mathrm{PO}_{4}$ (anhydrous) $2 \mathrm{~g}$, lactose $5 \mathrm{~g}$, maltose $3 \mathrm{~g}$, sodium linoleate $0 \cdot 2 \mathrm{~g}$, sodium citrate $4 \mathrm{~g}$, sodium thiosulphate (anhydrous) $4 \mathrm{~g}$, agar $15 \mathrm{~g}$, sodium deoxycholate $7 \mathrm{~g}$, bromocresol purple $0 \cdot 01 \mathrm{~g}$, neutral red $0 \cdot 02 \mathrm{~g}$, Tween $800 \cdot 25 \mathrm{~g}$, leucomycin $* 0.005 \mathrm{~g}$, and amphotericin $\mathrm{B}^{*}$ (Squibb) $0.01 \mathrm{~g}$. All components except those marked * were dissolved by heating to boiling point. The $p \mathrm{H}$ of the medium was adjusted to 7.0 with $\mathrm{NaOH}$ or $\mathrm{HCl}$. After cooling the medium to $c .60^{\circ} \mathrm{C}$, the selective agents $\left(^{*}\right)$ were added, and the medium poured into plates. (The leucomycin was first dissolved in absolute ethyl alcohol.) On these agar plates, $S$. typhimurium grew well, and $V$. parahaemolyticus and $Y$. enterocolitica grew moderately well. $E$. coli formed a few colonies on these agar plates when inoculated heavily $\left(10^{7} \mathrm{cfu} / \mathrm{plate}\right)$, but the colonies formed could be distinguished easily from those of Sh. flexneri.

\section{Results}

Population levels of enteropathogens in single or mixed cultures in MCM medium

The results are shown in figs. $2 \mathrm{~A}-\mathrm{F}$. The resident bacteria, E. coli, Ent. faecalis, B. ovatus, and $F$. varium, persisted at levels of $c .10^{6} \mathrm{cfu} / \mathrm{ml}$ or more in any mixed culture. Bif. adolescentis also persisted at the same level but only in the absence of $E$. coli (data not shown).

$Y$. enterocolitica was the most susceptible among the enteropathogens tested to antagonistic effects (fig. 2A). This organism persisted at a level of $c$. $5 \times 10^{6} \mathrm{cfu} / \mathrm{ml}$ in single culture but disappeared from the culture vessel within 5-6 days after inoculation in the presence of each of the four resident bacteria, E. coli, Ent. faecalis, B. ovatus, and $F$. varium.

Staph. aureus was excluded in the presence of $E$. coli alone (fig. 2B). Growth of Staph. aureus was strongly suppressed by Ent. faecalis but the staphy- lococcus persisted at a lower level of $c .10^{2} \mathrm{cfu} / \mathrm{ml}$. The numbers of Staph. aureus showed only c. 10fold reduction even in the presence of three resident bacteria, B. ovatus, $F$. varium, and Bif. adolescentis, as compared with the population level in single culture.

Sh.flexneri was excluded in the presence of E. coli (fig. 2C). In the presence of Ent. faecalis, B. ovatus, $F$. varium, and Bif. adolescentis, the numbers of $S h$. flexneri showed only $c .10^{2}$-fold reduction.

In contrast to the above three enteropathogens, $C$. perfringens (fig. 2D), $V$. parahaemolyticus (fig. $2 \mathrm{E}$ ), and $V$. cholerae (fig. $2 \mathrm{~F}$ ) were not excluded in the presence of any single resident strain, but $E$. coli ATCC 25922 showed the most potent antagonistic effect against them. Even when co-cultivated with this organism, populations of $C$. perfringens, $V$. parahaemolyticus, or $V$. cholerae showed only c. $10^{3}-, 10^{4}$-, and $<10$-fold reductions, respectively.

$C$. perfringens was excluded when co-cultivated with $E$. coli ATCC 25922 and $B$. ovatus. But in the presence of $E$. coli ATCC 11775 and $B$. ovatus, the population level of $C$. perfringens showed only $c$. 10fold reduction. In this case, two additional bacteria, Ent. faecalis and $F$. varium, were required to exclude $C$. perfringens. Three clinical isolates of $E$. coli showed rather weak antagonism comparable with that of E. coli ATCC 11775.

To exclude $V$. parahaemolyticus, co-cultivation with both $E$. coli ATCC 25922 and $B$. ovatus was also essential. In this case, identical results were obtained when $B$. ovatus was substituted with $B$. fragilis or $B$. thetaiotaomicron. A combination of $E$. coli ATCC 11775 and $B$. ovatus had only a minor effect on $V$. parahaemolyticus and even with the further addition of Ent. faecalis and $F$. varium, the population showed only $c$. 10 -fold reduction.

$V$. cholerae serogroup non 01 showed essentially the same responses as those of $V$. parahaemolyticus.

$S$. typhimurium was the most resistant of the enteropathogens tested to the antagonistic effects of the resident bacteria. In the presence of $E$. coli ATCC $25922, B$. ovatus, $F$. varium, and Ent. faecalis, the population level of this organism showed only $c .10^{2}$-fold reduction. In the presence of human faecal flora, the population level showed $c$. $10^{5}$-fold reduction and $S$. typhimurium persisted at c. $10^{3} \mathrm{cfu} / \mathrm{ml}$ (data not shown).

Effect of increase in nutritional components on population levels of two enteropathogens

The effects of an increase in components of the MCM medium on the populations of Sh. flexneri and $C$. perfringens, co-cultivated with five resident 

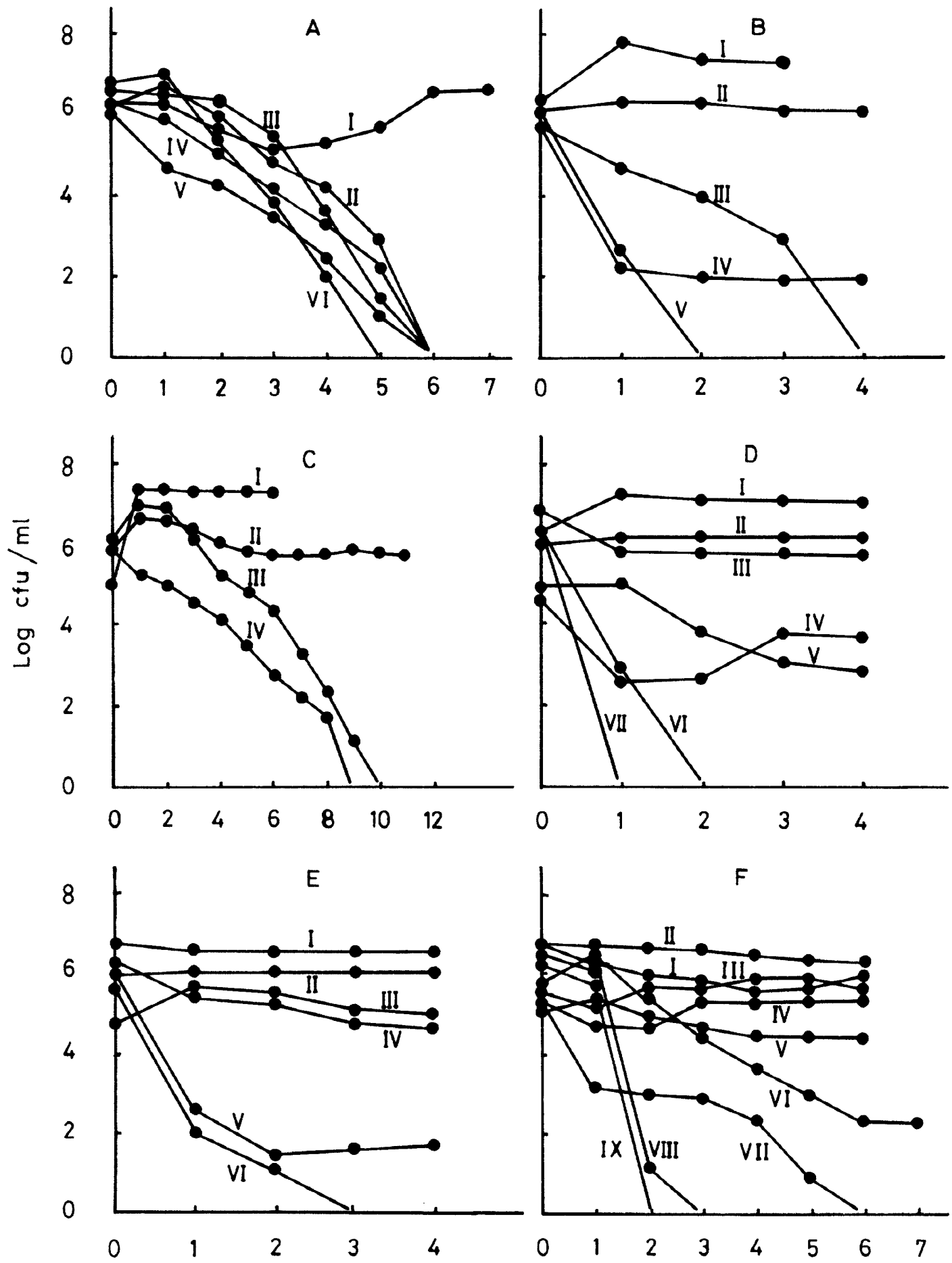

Days after inoculation

Fig. 2. Population levels of six entero pathogens in anaerobic CF cultures. Medium was MCM. Flow rate was $5 \% / \mathrm{h}(5 \mathrm{ml} / \mathrm{h})$. Final $\mathrm{pH}$ values of the culture media were in the range from $c .6 \cdot 5$ to 7.0 .

A: Y. enterocolitica SU 5032. I: Single culture; II: mixed culture with F. rarium; III: with E. coli ATCC 11775; IV: with Ent. faecalis; V: with E. coli ATCC 25922; VI: with B. ovatus.

B: Staph. aureus ATCC 25923. I: Single culture; II: mixed culture with B. ovatus, F. varium, and Bif. adolescentis; III: with E. coli ATCC 11775; IV: with Ent. faecalis; V: with E. coli ATCC 25922. 


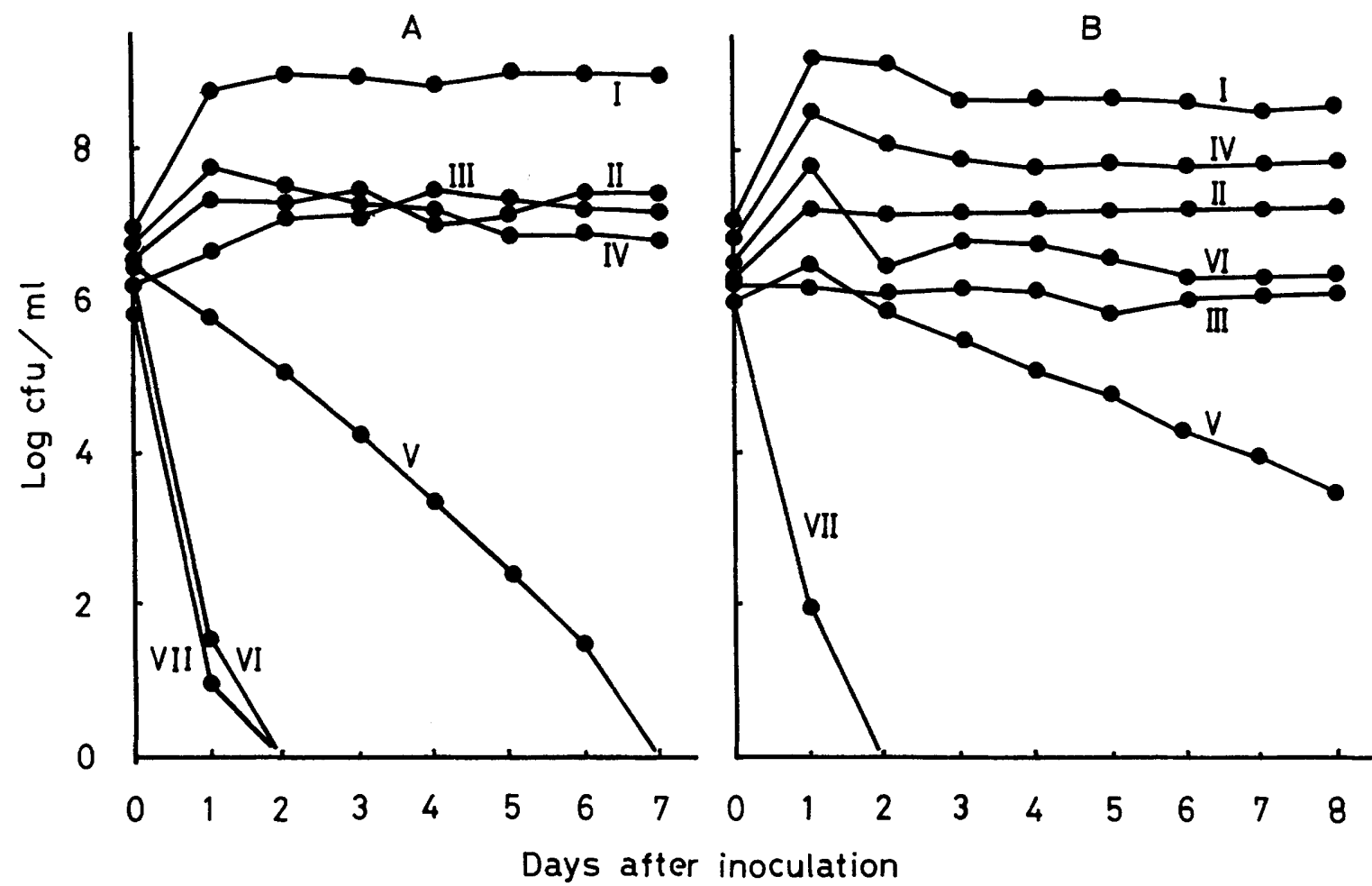

Fig. 3 Efect of concentration of some components in medium on population levels of seven bacterial strains (two enteropathogens and five resident bacteria of human colon) co-cultivated in anaerobic CF cultures.

A: Population levels of bacteria in original MCM medium. I: B. ovatus; II: E. coli ATCC 25922; III: F. varium; IV: Ent. faecalis; V: Sh. flexneri; VI: C. perfringens; VII: Bif. adolescentis.

B: Population levels in MCM medium enriched with peptone, yeast extract and starch. Concentration of proteose peptone No. 3, yeast extract, and starch was increased from $0.05 \%$ to $0 \cdot 5 \%, 0 \cdot 01 \%$ to $0.3 \%$, and $0 \cdot 2 \%$ to $0.5 \%$, respectively. Symbols: see legends to fig. 3-A.

bacteria, E. coli, Ent. faecalis, B. ovatus, F. varium, and Bif. adolescentis, are shown in figs. 3A-B.

In MCM medium (fig. 3A), B. ovatus was predominant at $c .5 \times 10^{9} \mathrm{cfu} / \mathrm{ml}$ among the resident bacteria used. The other three resident bacteria, $E$. coli, $F$. varium and Ent. faecalis, persisted at $10^{2}$ $10^{3}$-fold lower levels than that of $B$. ovatus. In contrast, Bif. adolescentis, C. perfringens, and Sh. flexneri were excluded.

When the concentration of Proteose Peptone No.
3 was increased from $0.05 \%$ to $0.5 \%, C$. perfringens persisted at a level of $c .10^{4} \mathrm{cfu} / \mathrm{ml}$. Identical results were obtained when the concentrations of glutamic acid, threonine and serine were increased from $0.03 \%$ to $0.3 \%, 0.004 \%$ to $0.05 \%$, and $0.001 \%$ to $0.05 \%$, respectively, in mixed culture with $B$. ovatus and $E$. coli ATCC 25922 (data not shown).

Further increases of two components, yeast extract from $0.01 \%$ to $0.3 \%$ and starch from $0.2 \%$ to $0.5 \%$, greatly increased the numbers of $C$.

C: Sh. flexneri 3a SU 5031. I: Single culture; II: mixed culture with B. ovatus, F. varium, Bif. adolescentis, and Ent. faecalis; III: with E. coli ATCC 25922; IV: with E. coli ATCC 11775.

D: C.perfringens VPI 5201. I: Single culture; II: mixed culture with B. ovatus; III: with E. coli ATCC 11775 and B. ovatus; IV: with $E$. coli ATCC 25922; V: with B. ovatus, f. varium, and Ent. faecalis; VI: with E. coli ATCC 11775, Ent. faecalis, B. ovatus and F. varium; VII: with $E$. coli ATCC 25922 and $F$. ovatus.

E: $V$. parahaemolyticus SU 5027. I: Single culture; II: mixed culture with $B$. ovatus, $F$. varium, and Ent. faecalis; III: with E. coli ATCC 11775 and B. ovatus; IV: with E. coli ATCC 11775, Ent. faecalis, B. ovatus, and F. varium; V: with E. coli ATCC 25922; VI: with E. coli ATCC 25922 and B. ovatus.

F: V. cholerae non O1 SU 5030. I: Single culture; II: Mixed culture with B. ovatus; III: with F. varium, Ent. faecalis, and E. coli ATCC 25922; IV: with E. coli ATCC 25922; V: with E. coli ATCC 11775 and B. ovatus; VI: with E. coli ATCC 11775, Ent. faecalis, B. ovatus and $F$. varium; VII: with $E$. coli ATCC 25922 and $B$. ovatus; VIII: with $E$. coli ATCC 25922 , B. ovatus and $F$. varium; IX: with $E$. coli ATCC 25922, B. ovatus and Ent. faecalis. 
perfringens to $c .10^{6} \mathrm{cfu} / \mathrm{ml}$ (fig. 3B). This population level was identical to that of the single culture in the original MCM medium (fig. 2D). Even with this enriched medium, there was a steady reduction of the population of Sh.flexneri, but the reduction rate was reduced to $c$. one-third of that in the original MCM medium. Bif. adolescentis was readily excluded, even in this enriched medium.

\section{Discussion}

There are reports on the potent unilateral antagonism of $E$. coli, alone or in combination with other bacterial species such as Ent. faecalis, against $S h$. flexneri in anaerobic CF cultures or in the alimentary canal of gnotobiotic animals (Hentges and Freter. 1962; Maier et al., 1972; Tazume et al., 1979). In contrast. data on interactions between normal faecal flora and enteropathogens other than Sh. flexneri are few (Ransom et al., 1961).

In the present study, we confirmed the antagonism of $E$. coli against Sh. flexneri, and we also found that four resident bacteria of the human colon. E. coli, Ent faecalis, F. rarium, and B. oratus, alone or in combination, were antagonistic not only to Sh.flexneri but also to several other enteropathogens. In some cases the $B$. oratus component could be substituted by $B$. fragilis or $B$. thetaiotaomicron. $E$. coli had the predominant antagonistic activity.

As already reported by Hentges and Freter (1962). E. coli strains vary in their antagonistic potency. E. coli ATCC 25922, for example, is more active than $E$. coli ATCC 11775 . To exclude or reduce the population of some enteropathogens, strain ATCC 25922 required the cooperation of only $B$. oratus whereas strain ATCC 11775 required that of B. ovatus plus Ent. faecalis or F. varium or both. These results indicate synergic antagonistic activities among the resident bacteria of the human colon.

Freter et al. (1983b) indicated that even in simplified studies with synthetic flora, the population levels attained by co-existing bacteria in anaerobic CF cultures correlated well with those noted in the mouse caecum. They speculated that similar mechanisms may operate naturally to control population levels of bacteria in these ecosystems. Accordingly, we speculate that the antagonism exhibited by resident bacteria of the colon against several enteropathogens in our anaerobic CF cul- tures may be relevant to control systems that operate in the lower alimentary canal of man.

The effects of changes in concentrations of nutritional components on the population levels of two enteropathogens are also of interest. Sh. flexneri was reduced steadily in both the original and the enriched MCM medium, whereas $C$. perfringens grew and persisted at a level of $c .10^{4} \mathrm{cfu} / \mathrm{ml}$ in MCM medium enriched with peptone. Identical results were obtained when additional amounts of glutamic acid, threonine and serine were used instead of additional peptone, in the mixed culture with $E$. coli and $B$. ovatus.

As we observed in the anaerobic CF culture, Månnson et al. (1971) found great increases (to c. $10^{7}-10^{8} \mathrm{cfu} / \mathrm{g}$ ) in the population of $C$. perfringens in faeces of pigs fed a protein-rich diet. In the control pigs fed a normal diet, $C$. perfringens could not be demonstrated or was at very low levels of $c .10^{2} \mathrm{cfu} /$ $\mathrm{g}$ of faeces or less.

Freter et al. (1983a) postulated that each of the several hundred bacterial species that reside in the alimentary canal is controlled by one or a few nutritional components which can be utilised most efficiently in the presence of many inhibitory substances such as $\mathrm{H}_{2} \mathrm{~S}$ and fatty acids, and under conditions of $p \mathrm{H}$ and anaerobiosis prevailing in the canal. In the case of $C$. perfringens, nitrogen sources are one of the population-controlling components.

The population level of $C$. perfringens in faeces varies considerably between individuals, the range being from $10^{1}$ to $10^{9} \mathrm{cfu} / \mathrm{g}$ (Akama and Otani, 1970), and varies widely in each individual (Akama et al. 1966). One of the associated factors may be the changes in amounts of available nutrients such as nitrogen sources moving down into the lower alimentary canal.

Bif. adolescentis is one of the predominant residents in the human colon. In anaerobic CF cultures, this organism was usually promptly excluded in the presence of $E$. coli (data not shown). The failure of persistence of Bif. adolescentis in CF cultures, even in enriched media, seems to be due to the high $p \mathrm{H}$ value of the culture media ( $\mathrm{pH} 6.5$ to 7.0 ) which does not allow rapid growth of this organism (Edwards et al., 1985), especially in the presence of potent antagonistic bacteria such as $E$. coli.

Thanks are due to $\mathrm{M}$. Ohara for comments on the manuscript. 


\section{REFERENCES}

Akama K, Otani S 1970 Clostridium perfringens as the flora in the intestine of healthy persons. Japanese Journal of Medical Science and Biology 23: 161-175.

Akama $\mathrm{K}$ et al. 1966 Studies on Clostridum welchii as one of the bacterial flora of normal human faeces. I. Bacterial counts of total and heat-resistant organisms. Japanese Journal of Bacteriology 21:619-625. (in Japanese)

Edwards C A, Duerden B I, Read N W 1985 The effects of $p \mathrm{H}$ on colonic bacteria grown in continuous culture. Journal of Medical Microbiology 19:169-180.

Freter R, Brickner H, Botney M, Cleven D, Aranki A 1983a Mechanisms that control bacterial populations in continuous-flow culture models of mouse large intestinal flora. Infection and Immunity 39:676-685.

Freter R, Stauffer E, Cleven D, Holdeman L V, Moore W E C $1983 b$ Continuous-flow cultures as in vitro models of the ecology of large intestinal flora. Infection and Immunity 39:666-675.

Hentges D J, Freter R 1962 In vivo and in vitro antagonism of intestinal bacteria against Shigella flexneri I. Correlation between various tests. Journal of Infectious Diseases 110:30-37.

Maier B R, Onderdonk A B, Baskett R C, Hentges D J 1972 Shigella, indigenous flora interactions in mice. American Journal of Clinical Nutrition 25: 1433-1440.

Månsson I, Norberg R, Olhagen B, Björklund N E 1971 Arthritis in pigs induced by dietary factors. Microbiologic, clinical and histologic studies. Clinical and Experimental Immunology 9:677-693.

Parker C A 1955 Anaerobiosis with iron wool. Australian Journal of Experimental Biology and Medical Science 33:33-37.
Ransom J P, Finkelstein R A, Ceder R E, Formal S B 1961 Interactions of Vibrio cholerae, Shigella flexneri, enterococci and lactobacilli in continuously fed cultures. Proceedings of the Society for Experimental Biology and Medicine 107:332336.

Tazume S, Hashimoto K, Sasaki S 1979 Intestinal flora and bile acids metabolism. Influence of bile acids on rejection of Shigella flexneri $2 \mathrm{a}$ from the intestine. Japanese Journal of Bacteriology 34:745-754. (in Japanese)

Ushijima T $1983 a$ Semisynthetic media for in vitro examination of the contributions of nutrients and growth inhibitors for symbiosis or antagonism of the bacteria in ileum, caecum and colon of the mammalian. Igaku to Seibutsugaku (Medicine and Biology) 106: 199-204. (in Japanese).

Ushijima T, Takahashi M, Tatewaki K, Ozaki Y $1983 b$ A selective medium for isolation and presumptive identification of the Bacteroides fragilis group. Microbiology and Immunology 27:985-993.

Ushijima T 1984 A simple anaerobic continuous culture system for the study of bacterial symbiosis and antagonism. Igaku to Seibutsugaku (Medicine and Biology) 108:71-74. (in Japanese).

Ushijima T, Takahashi M, Ozaki Y 1985 Fourteen selective media facilitate evaluation of populations of coexisting fixed bacterial strains of enteric pathogens and normal human fecal flora. Journal of Microbiological Methods 4:189-194.

Veilleux B G, Rowland I 1981 Simulation of the rat intestinal ecosystem using a two-stage continuous culture sytem. Journal of General Microbiology 123: 103-115. 